Journal for Educational Research Online

Journal für Bildungsforschung Online

https://doi.org/10.31244/jero.2021.02.04

Volume 13 (2021), No. 2, 62-83

CC BY-NC-SA 4.o Waxmann 2021

Sebastian Thürer*, Nanine Lilla*, Wim Nieuwenboom

\& Marianne Schüpbach

\title{
Individuelle und im Klassenkontext \\ vorherrschende Akkulturationsorientierung und die individuelle Lesekompetenz von Schülerinnen und Schülern der 9. Klasse
}

\section{Zusammenfassung}

Im Fokus dieser Querschnittstudie steht die individuelle und im Klassenkontext vorherrschende Akkulturationsorientierung und der Zusammenhang mit der erreichten Lesekompetenz von Schülerinnen und Schülern der 9. Klasse. Akkulturationsorientierung wird hierbei im Sinne Berrys (1997) als individuelle Orientierung an der Aufnahme- und Herkunftskultur verstanden, welche in dieser Studie anhand latenter Profilanalysen unter Berücksichtigung affektiver, behavioraler und kognitiver Aspekte komplex erfasst wird. Die so ermittelten Akkulturationsprofile, assimiliert, integriert, separiert und indifferent, werden in Mehrebenenanalysen zunächst auf individueller Ebene berücksichtigt, um die Lesekompetenz von Schülerinnen und Schülern mit Migrationshintergrund in Abhängigkeit von ihren Akkulturationsprofilen mit der von Schülerinnen und Schülern ohne Migrationshintergrund erreichten Lesekompetenz zu vergleichen. Weiter werden die im Klassenkontext vorherrschenden Akkulturationsprofile auf Klassenebene modelliert. Auf Individualebene zeigt sich, dass Schülerinnen und Schüler mit separiertem und indifferentem Akkulturationsprofil niedrigere Lesekompetenzen erzielen als Schülerinnen und Schüler ohne Migrationshintergrund, auf Klassenebene stehen die Anteile der unterschiedlichen Akkulturationsprofile in der Klasse nach Berücksichtigung des besuchten Schultyps in keinem Zusammenhang mit der Lesekompetenz der Schülerinnen und Schüler.

\footnotetext{
* geteilte Erstautor:innenschaft

Sebastian Thürer (Korrespondenzautor) · Dr. Nanine Lilla · Dr. Wim Nieuwenboom · Prof. Dr. Marianne Schüpbach, Arbeitsbereich Allgemeine Grundschulpädagogik, Freie Universität Berlin, Habelschwerdter Allee 45, 14195 Berlin

E-Mail: sebastian.thuerer@fu-berlin.de nanine.lilla@fu-berlin.de wim.nieuwenboom@fu-berlin.de marianne.schuepbach@fu-berlin.de
} 


\title{
Schlagworte
}

Latente Profilanalyse, Schülerinnen und Schüler mit Migrationshintergrund, Klassenkomposition, Bildungsungleichheit, Akkulturationsorientierung

\section{Individual Acculturation Orientation and Prevailing Acculturation Orientation in Class and Their Association with Individual Reading Competence of Ninth Grade Students}

\begin{abstract}
The focus of this cross-sectional study is the individual acculturation orientation and the acculturation orientation prevailing in the classroom context and their association with the reading competence of ninth grade students. Acculturation orientation is understood in the sense of Berry (1997) as the individual's orientation towards the host culture and towards the culture of origin, which in this study is identified in a complex way using latent profile analyses considering affective, behavioral and cognitive aspects. The acculturation profiles identified, assimilated, integrated, segregated, and indifferent, are first considered on the individual level in multilevel analyses to compare the reading competence of students with a migrant background as a function of their acculturation profile with the reading competence achieved by students without a migrant background. Furthermore, the acculturation profiles prevailing in the classroom context are modelled at the class level. At the individual level, results show that students with separated and indifferent acculturation profile achieve lower reading competences than students without a migrant background; at the class level, the proportions of the different acculturation profiles in class show not to be related to the reading competences of the students after the type of school attended has been considered.
\end{abstract}

\section{Keywords}

latent profile analysis, students with migrant background, class composition, educational inequality, acculturation orientation

\section{Einleitung}

Trotz vielfältiger Anstrengungen seit dem „PISA-Schock“ vor über zwei Jahrzehnten die teils gravierenden Unterschiede in den schulischen Leistungen zwischen Schülerinnen und Schülern mit und ohne Migrationshintergrund in Deutschland zu minimieren, bestehen diese weiter fort. So wurde in der aktuellen Erhebung des Programme for International Student Assessment (PISA) festgestellt, dass beispielsweise in der Lesekompetenz eine Differenz von durchschnittlich 52 Punkten zwischen Schülerinnen und Schülern mit Migrationshintergrund (472 Punkte) und Schülerinnen und Schülern ohne Migrationshintergrund (524 Punkte) besteht, 
was einem Leistungsrückstand von etwa zwei Schuljahren entspricht (Weis et al., 2019). Gleichzeitig steigen in den Schulklassen des deutschen Bildungssystems aus Gründen wie beispielsweise Arbeits- oder Fluchtmigration die Anteile der Schülerinnen und Schüler mit Migrationshintergrund, das heißt der Schülerinnen und Schüler, die selbst nicht in Deutschland geboren wurden oder mindestens einen Eltern- oder Großelternteil haben, der nach Deutschland zugewandert ist (Autorengruppe Bildungsberichterstattung, 2018).

In der empirischen Bildungsforschung werden die Bildungsdiskrepanzen zwischen Schülerinnen und Schülern mit und ohne Migrationshintergrund vielfach auf primäre und sekundäre Herkunftseffekte zurückgeführt (Boudon, 1974). So bewirken einerseits Unterschiede in der familiären Ressourcenausstattung und andererseits die damit in Zusammenhang stehenden Bildungsentscheidungen seitens der Eltern Bildungsungleichheiten zwischen Schülerinnen und Schülern mit und ohne Migrationshintergrund (Becker, 2011; Dumont, Maaz, Neumann \& Becker, 2014). Studien zur Klassen- oder Schulkomposition zeigen überdies, dass die Kumulierung bestimmter Schülerinnen- und Schülermerkmale in Schulklassen oder Schulen die Leistung aller Schülerinnen und Schüler der entsprechenden Schulklasse oder Schule beeinflusst (Dumont, Neumann, Maaz \& Trautwein, 2013).

Neben Merkmalen, die als primäre und sekundäre Herkunftseffekte wirken, spielen auch psychologische Variablen eine Rolle bei der Erklärung des Schulerfolgs von Schülerinnen und Schülern mit Migrationshintergrund. Ein Beispiel stellt die Akkulturationsorientierung der Schülerinnen und Schüler mit Migrationshintergrund dar, welche nach Berry (1997) definiert ist durch die Orientierung an der Kultur des Aufnahmelands und die Orientierung an der Kultur des Herkunftslands. Die wenigen für Deutschland vorliegenden Studien zeigen, dass insbesondere die Schülerinnen und Schüler, die sich stärker an der deutschen Kultur orientieren und sich stärker mit der deutschen Gesellschaft identifizieren, bildungserfolgreicher sind (Edele, Stanat, Radmann \& Segeritz, 2013; Hannover et al., 2013; Schotte, Stanat \& Edele, 2018). Dabei wurden die Akkulturationsorientierungen von Schülerinnen und Schülern mit Migrationshintergrund für den Vergleich mit Schülerinnen und Schülern ohne Migrationshintergrund im Hinblick auf Schulleistung bislang in nur einer der Studien in den Blick genommen (Edele et al., 2013). Inwiefern die Kumulierung von Akkulturationsorientierungen auf Klassenoder Schulebene im Zusammenhang mit der Schulleistung aller Schülerinnen und Schüler einer Schulklasse steht, wurde unseres Wissens bislang nicht untersucht.

Daran knüpft die vorliegende Studie an, die auf Basis der Daten des Nationalen Bildungspanels (NEPS; Blossfeld, Roßbach \& von Maurice, 2011) in einem querschnittlichen Design die erreichte Lesekompetenz von Neuntklässlerinnen und Neuntklässlern mit Migrationshintergrund in Abhängigkeit von ihren Akkulturationsorientierungen vergleicht mit der Lesekompetenz von Schülerinnen und Schülern ohne Migrationshintergrund und Zusammenhänge zwischen den Anteilen der in der Schulklasse vorherrschenden Akkulturationsorientierungen und der Lesekompetenz aller Schülerinnen und Schüler einer Schulklasse untersucht. In Erweiterung des aktuellen deutschsprachigen Forschungsstands werden die Akkul- 
turationsorientierungen der Schülerinnen und Schüler mit Migrationshintergrund anhand eines umfassenden Indikators, der sowohl affektive, behaviorale als auch kognitive Aspekte der individuellen Akkulturation berücksichtigt, komplex erfasst.

\section{Theoretischer und empirischer Hintergrund}

\subsection{Akkulturationsorientierung und schulische Adaption}

Menschen mit Migrationshintergrund befinden sich im Spannungsverhältnis zwischen der Kultur des Aufnahmelandes und der Kultur des Herkunftslandes. Durch diesen Umstand erfolgt ein Prozess der Akkulturation, aus welchem sich unterschiedliche Möglichkeiten der kulturellen Verortung ergeben. Grundsätzlich, so Berry (Berry, 1997; Berry \& Hou, 2016; Berry, Kim, Power, Young \& Bujaki, 1989), vollzieht sich die Orientierung auf zwei voneinander unabhängigen Dimensionen: der Orientierung an der Kultur des Aufnahmelandes und der Orientierung an der Kultur des Herkunftslandes. Theoretisch lassen sich so vier Akkulturationsorientierungen unterscheiden: Integration, wenn sowohl die Orientierung an der Kultur des Aufnahme- als auch an der Kultur des Herkunftslandes stark ausgeprägt ist; Assimilation ergibt sich aus einer starken Orientierung an der Kultur des Aufnahmelandes bei gleichzeitiger schwacher Orientierung an der Kultur des Herkunftslandes; Separation, wenn die Orientierung an der Kultur des Aufnahmelands schwach, gleichzeitig aber die Orientierung an der Kultur des Herkunftslands stark ausfällt. Ist sowohl die Orientierung an der Kultur des Aufnahme- als auch an der Kultur des Herkunftslandes schwach ausgeprägt, wird dies als Marginalisierung bezeichnet.

Unterschiedliche Akkulturationsorientierungen lassen unterschiedliche Akkulturationsoutcomes erwarten, wobei Ward (1996) zwischen psychologischer und soziokultureller Adaption unterscheidet. Allgemein postuliert Berry (1997), dass Integration als vorteilhafteste Akkulturationsorientierung betrachtet werden kann und begründet dies mit der breiten Zugänglichkeit zu Ressourcen, sowohl aus dem Aufnahmekontext als auch aus dem Herkunftskontext. Entsprechend wird Marginalisierung als diejenige Akkulturationsorientierung, die die wenigsten Ressourcen bereithält, als am wenigsten adaptiv vermutet. Da Assimilation und Separation aufgrund ihrer starken Orientierung an nur einer Kultur den Zugriff auf je nur einen Ressourcenkontext ermöglichen, bezeichnet Berry (1997) diese als mittelmäBige Optionen.

Der Erwerb schulischer Kompetenzen stellt ein wichtiges Akkulturationsoutcome von Schülerinnen und Schülern mit Migrationshintergrund dar. Ein systematisches Review - vornehmlich US-amerikanischer Studien - verweist auf studienübergreifend positive Zusammenhänge zwischen einer nationalen Orientierung und dem Erwerb schulischer Kompetenzen, findet jedoch kein eindeutiges Befundmuster für den Zusammenhang zwischen Schulerfolg und ethnischer Orientierung, d.h. einer Orientierung an der Herkunftskultur (Makarova \& Birman, 2015). 
Dasselbe Muster zeigen auch die Befunde von Schotte, Stanat und Edele (2018), die auf Grundlage von Daten des NEPS untersuchten, inwieweit im deutschen Schulkontext die kulturelle Identifikation mit dem Aufnahme- bzw. mit dem Herkunftsland in Zusammenhang mit der Schulleistung von Schülerinnen und Schülern mit Migrationshintergrund stehen. Die Ergebnisse zeigen durchweg signifikant positive Zusammenhänge zwischen der Identifikation mit dem Aufnahmeland und den Noten in Deutsch und Mathematik sowie den Lese- und Mathematikkompetenzen. Ein signifikant negativer Zusammenhang zeigt sich hingegen zwischen der Identifikation mit dem Herkunftsland und den Mathematikkompetenzen, während zwischen der Identifikation mit dem Herkunftsland und den Noten in Deutsch und Mathematik sowie den Lesekompetenzen keine Zusammenhänge zu bestehen scheinen. Darüber hinausgehend konnten Schotte et al. (2018) für keine der Schulleistungsvariablen einen Benefit einer gleichzeitig starken Identifikation mit dem Aufnahmeland und mit dem Herkunftsland zeigen, was Berrys Annahme einer Überlegenheit der Integration gegenüber der Assimilation nicht stützt.

Ähnlich zeigte sich in einer weiteren deutschen Studie von Hannover et al. (2013) beim Vergleich der Lesekompetenzen zwischen Schülerinnen und Schülern mit Migrationshintergrund mit unterschiedlichen kulturellen Selbstbildern im Schulkontext nur ein signifikanter Unterschied zwischen Schülerinnen und Schülern, deren Selbstbeschreibung sich ausschließlich auf ihre Herkunft bezog, und Schülerinnen und Schülern, deren Selbstbeschreibung als assimiliert interpretiert werden kann, nicht aber im Vergleich zu Schülerinnen und Schülern, deren Selbstbeschreibung als integriert interpretiert werden kann. Außerdem erwies sich in dieser Studie, in der die geclusterte Datenstruktur in einem Mehrebenenmodell berücksichtigt wurde, der besuchte Schultyp als stärkster Prädiktor der Lesekompetenz.

Bis anhin wurden weitgehend die Kompetenzen von Schülerinnen und Schülern mit Migrationshintergrund in Abhängigkeit ihrer unterschiedlichen Akkulturationsorientierungen miteinander verglichen. Nach unserem Wissen hat lediglich eine Studie im deutschen Kontext den Vergleich dieser mit den Kompetenzen von Schülerinnen und Schülern ohne Migrationshintergrund vorgenommen. Auf Grundlage der PISA-Daten von 2009 untersuchten Edele, Stanat, Radmann und Segeritz (2013) die Lesekompetenzen von Schülerinnen und Schülern mit Migrationshintergrund in Abhängigkeit ihrer Akkulturationsorientierungen im Vergleich zu den Lesekompetenzen von Schülerinnen und Schülern ohne Migrationshintergrund. Die vier Akkulturationsorientierungen sensu Berry (1997) wurden in dieser Studie über eine Dichotomisierung und Kreuzung der beiden Skalen Identifikation mit der Aufnahmegesellschaft und Identifikation mit der Herkunftsgesellschaft operationalisiert. Unter Kontrolle relevanter Hintergrundmerkmale wurde gezeigt, dass assimilierte Schülerinnen und Schüler mit Migrationshintergrund gegenüber Schülerinnen und Schülern ohne Migrationshintergrund höhere Werte bei den Kompetenzmessungen im Lesen erreichen. Für Schülerinnen und Schüler mit der Akkulturationsorientierung Integration zeigte sich hingegen kein signifikanter Unterschied im Vergleich zu Schülerinnen und Schülern ohne Migrationshinter- 
grund. Separierte und marginalisierte Schülerinnen und Schüler zeigten gegenüber ihren Mitschülerinnen und Mitschülern ohne Migrationshintergrund eine deutlich geringere Leseleistung, die zumindest für die Separierten aber auf die in der Familie gesprochene Sprache zurückgeführt werden konnte (Edele et al., 2013). Auch diese Ergebnisse sprechen nicht für eine Überlegenheit der Integration gegenüber der Assimilation und zeigen darüber hinaus die starke Adaptivität der Assimilation im deutschen Schulkontext, die im Vergleich zu Schülerinnen und Schülern ohne Migrationshintergrund sogar mit besseren Lesekompetenzen einherzugehen scheint.

Zusammengefasst zeigt der Vergleich mit Schülerinnen und Schülern ohne Migrationshintergrund, dass für Schülerinnen und Schüler mit assimilierter und integrierter Akkulturationsorientierung keine negative Bildungsdiskrepanz besteht, sondern die Bildungsungleichheit zwischen Schülerinnen und Schülern mit und ohne Migrationshintergrund lediglich auf die separierten und marginalisierten Schülerinnen und Schüler zurückgeführt werden muss. Die Validität dieser Befunde ist jedoch insofern eingeschränkt, als weder die im Schulkontext vorherrschende hierarchische Datenstruktur (Schülerinnen und Schüler in Klassen) noch der Einfluss der besuchten Schulform berücksichtigt wurde.

\subsection{Empirische Erfassung der Akkulturationsorientierung}

Berrys (1997) bidimensionale Betrachtung der Akkulturationsorientierung folgt der Auffassung, dass mit einer starken Orientierung an einer Kultur nicht zwingend eine schwache Orientierung beziehungsweise ein Verlust der anderen Kultur einhergehen muss, sondern beide Orientierungen koexistent sein können. Zudem erfolgt Akkulturation u. a. nach Berrys Auffassung nicht ausschließlich auf Ebene der Identifikation, sondern beinhaltet Veränderungen affektiver, verhaltensbezogener und kognitiver Aspekte (Schwartz, Unger, Zamboanga \& Szapocznik, 2010).

Unter Verwendung von Daten über kulturelle Identität, Einstellungen und Werte, soziale Beziehungen, Sprachkenntnis und Sprachgebrauch von über $4000 \mathrm{Ju}-$ gendlichen aus dreizehn Nationen konnten Berry, Phinney, Sam und Vedder (2006) anhand einer Clusteranalyse die empirische Existenz von vier distinkten Clustern (integration, national, ethnic, diffuse), welche inhaltlich weitestgehend den vier theoretisch angenommenen Akkulturationsorientierungen Integration, Assimilation, Separation und Marginalisierung entsprechen, zeigen.

Einen noch stärker datengeleiteten Ansatz für eine empirische Erfassung von Akkulturationsorientierungen unter Berücksichtigung unterschiedlicher mit Akkulturation im Zusammenhang stehender Variablen stellen latente Klassen- oder Profilanalysen dar (vgl. Schwartz \& Zamboanga, 2008). Je nach Stichprobe sowie berücksichtigten Variablen zeigen unterschiedliche Studien verschiedene Anzahlen von Akkulturationsklassen bzw. Akkulturationsprofilen auf, die den von Berry postulierten Akkulturationsorientierungen mehr oder weniger entsprechen, wobei Abweichungen häufig das Konzept der Marginalisierung in Frage stellen (Fox, Merz, 
Solórzano \& Roesch, 2013; Matsunaga, Hecht, Elek \& Ndiaye, 2010; Nieri, Lee, Kulis \& Marsiglia, 2011; Schwartz \& Zamboanga, 2008).

Für Deutschland zeigt eine auf Grundlage der Daten zur nationalen und ethnischen Identifikation von knapp 500 Jugendlichen mit türkischem Migrationshintergrund bzw. Herkunft aus der ehemaligen Sowjetunion durchgeführte Profilanalyse für die Gruppe der Türkischstämmigen vier unterschiedliche Akkulturationsprofile, in der Gruppe der Jugendlichen mit Aussiedler-Hintergrund wurden hingegen nur drei Akkulturationsprofile identifiziert (Jugert, Pink, Fleischmann \& Leszczensky, 2020). In beiden Gruppen konnte keine Evidenz für Marginalisierung gezeigt werden.

Beim Versuch, die vier theoretisch postulierten Akkulturationsorientierungen sensu Berry (1997) in einem deutschlandweit repräsentativen Datensatz von Schülerinnen und Schülern der 9. Klasse zu replizieren, ermittelten Lilla, Thürer, Nieuwenboom und Schüpbach (2021) mit Daten von über 1300 Schülerinnen und Schülern mit Migrationshintergrund der vier größten Herkunftsgruppen aus dem Datensatz der Startkohorte 4 des NEPS unter Berücksichtigung sowohl affektiver, behavioraler als auch kognitiver Aspekte vier latente Profile, wovon eines als integriertes, eines als assimiliertes und eines als separiertes Akkulturationsprofil interpretiert wurde sowie ein weiteres Akkulturationsprofil, welches jeweils nur in der Tendenz dem assimilierten Profil entsprach und in der Folge als moderat assimiliert beschrieben wurde. Ein marginalisiertes Akkulturationsprofil, welches sich durch eine relativ geringe Zustimmung in allen Skalen hätte ausdrücken müssen, zeigte die latente Profilanalyse nicht.

Verglichen mit den vorgestellten deutschsprachigen Studien (Edele et al., 2013; Hannover et al., 2013; Jugert et al., 2020; Schotte et al., 2018), die bislang vornehmlich auf den identifikativen Aspekt fokussierten, stellt eine komplexe Erfassung von Akkulturationsorientierungen, in die die konzeptionelle Mehrschichtigkeit von Akkulturation (Schwartz et al., 2010) Eingang findet, eine Erweiterung des Forschungsstands dar. Die Einschränkung auf identifikative Aspekte bei der Operationalisierung von Akkulturationsorientierungen ist insofern problematisch, als davon ausgegangen werden muss, dass unterschiedliche Ergebnisse erzielt werden, wenn Akkulturationsorientierungen basierend auf behavioralen, anstelle von affektiven, Merkmalen operationalisiert werden (Ward, 2001).

\subsection{Akkulturationsorientierung als Kompositionsmerkmal der Klasse}

Ähnlich wie angenommen wird, dass die individuelle Akkulturationsorientierung von Schülerinnen und Schülern mit Migrationshintergrund die Zugänglichkeit zu relevanten Ressourcen determiniert und darüber ein Zusammenhang mit schulischer Leistung besteht, basiert die Forschung zur Klassenkomposition unter anderem auf der Annahme, dass die Zusammensetzung der Schülerschaft einer Klasse die Interaktionsmöglichkeiten für alle Schülerinnen und Schüler und die Bandbrei- 
te an im Klassenzimmer verfügbaren potenziellen Ressourcen determiniert und sich auf die Leistung der Schülerinnen und Schüler niederschlägt (Driessen, 2002).

Entsprechend zeigt der Forschungsstand zur Klassenkomposition in Hinblick auf die Leistung von Schülerinnen und Schülern Zusammenhänge mit bestimmten auf Klassen- oder Schulebene aggregierten Individualmerkmalen wie beispielsweise der leistungsbezogenen Komposition oder der Zusammensetzung in Bezug auf den sozioökonomischen Hintergrund (siehe Dumont et al., 2013, für einen Überblick). Während internationale Studien auch Zusammenhänge mit der ethnischen Komposition belegen, lassen sich für Deutschland diesbezüglich keine spezifischen Effekte auf Schulleistung zeigen, die über die sozioökonomische Zusammensetzung hinausgehen (Stanat, 2006; Stanat, Schwippert \& Gröhlich, 2010).

Als Kompositionsmerkmal bislang weniger adressiert - abgesehen vom sozioökonomischen Status - wurden Hintergrundvariablen, die in Zusammenhang mit einem Migrationshintergrund der Schülerinnen und Schüler stehen können, wie beispielsweise eine andere Erstsprache als Deutsch. Seuring, Rjosk und Stanat (2020) können mit einer Querschnittsanalyse mit Daten des NEPS zeigen, dass der Anteil der Schülerinnen und Schüler mit einer anderen Erstsprache in der Klasse negativ mit der Lesekompetenz der Schülerinnen und Schüler der Klasse assoziiert ist und der Zusammenhang stärker ist als der zwischen dem Anteil der Schülerinnen und Schüler mit Migrationshintergrund in der Klasse und der Lesekompetenz. Auch wenn der gezeigte Zusammenhang im Vergleich zu anderen Faktoren gering ist und letztlich nur für die Schülerinnen und Schüler mit einer anderen Erstsprache gezeigt werden konnte, möchten wir diesen Ansatz weiterverfolgen und untersuchen, inwiefern die in der Klasse vorherrschenden Akkulturationsorientierungen als Kompositionsmerkmal in Zusammenhang mit der Schulleistung von Schülerinnen und Schülern stehen. Erste Studien, welche die Akkulturationsorientierung auf der Klassenebene berücksichtigen, beziehen sich auf deren Bedeutung im Zusammenhang mit sozialer Ausgrenzung im Schulkontext (Celeste, Meeussen, Verschueren \& Phalet, 2016).

Nach unserem Wissen wurden im deutschen Kontext keine Studien zu Akkulturationsorientierungen auf Klassenebene durchgeführt und Zusammenhänge mit Schulleistung bislang nicht in den Blick genommen.

\section{Fragestellungen}

Aufbauend auf dem dargestellten theoretischen und empirischen Hintergrund zielt die vorliegende Studie darauf ab, den bisherigen Forschungsstand zur Lesekompetenz von Schülerinnen und Schülern mit Migrationshintergrund in Abhängigkeit von ihrer Akkulturationsorientierung im Vergleich zur Lesekompetenz von Schülerinnen und Schülern ohne Migrationshintergrund zu erweitern, indem zum einen Akkulturationsorientierungen komplex erfasst werden und zum anderen die Mehrebenenstruktur der Daten sowie die besuchte Schulform berücksichtigt werden. Zudem werden die Akkulturationsorientierungen der Schülerinnen und Schüler mit 
Migrationshintergrund auf Klassenebene aggregiert und Zusammenhänge mit der individuellen Lesekompetenz der Schülerinnen und Schüler 9. Jahrgangsstufen in Deutschland untersucht. Aufbauend auf mittels latenter Profilanalyse unter Berücksichtigung affektiver, behavioraler und kognitiver Aspekte komplex erfasster Akkulturationsprofile wird den folgenden Fragestellungen nachgegangen.

1. Inwiefern unterscheidet sich die individuelle Lesekompetenz von Schülerinnen und Schülern mit Migrationshintergrund in Abhängigkeit von ihrem Akkulturationsprofil von der Lesekompetenz von Schülerinnen und Schülern ohne Migrationshintergrund?

Ausgehend vom aktuellen Forschungsstand im deutschen Schulkontext erwarten wir keine negativen Diskrepanzen in der Lesekompetenz für Schülerinnen und Schüler mit assimiliertem und integriertem Akkulturationsprofil im Vergleich zu Schülerinnen und Schülern ohne Migrationshintergrund. Für Schülerinnen und Schüler mit separiertem Akkulturationsprofil nehmen wir hingegen eine im Vergleich zu Schülerinnen und Schülern ohne Migrationshintergrund niedrigere Lesekompetenz an.

2. Inwiefern bestehen Zusammenhänge zwischen den im Klassenkontext vorherrschenden Akkulturationsprofilen und der individuellen Lesekompetenz der Schülerinnen und Schüler?

Aufgrund der fehlenden Studienlage leiten wir unsere Annahmen zu möglichen $\mathrm{Zu}-$ sammenhängen zwischen den Akkulturationsorientierungen auf Klassenebene als Kompositionsmerkmal und der individuellen Lesekompetenz der Schülerinnen und Schüler einer Klasse aus den Befunden bisheriger Studien auf Individualebene her. Folglich nehmen wir an, dass der Anteil von separierten Schülerinnen und Schülern mit Migrationshintergrund in einer Klasse in negativem Zusammenhang mit der Lesekompetenz der Schülerinnen und Schüler der Klasse steht.

\section{Methode}

\subsection{Stichprobe}

Als Ausgangslage für die Analysen dienen die Daten der Startkohorte 4 (SC 4) des Nationalen Bildungspanels (Blossfeld et al., 2011). ${ }^{1}$ Nach Ausschluss der Schüle-

1 Diese Arbeit nutzt Daten des Nationalen Bildungspanels (NEPS): Startkohorte Klasse 9, doi:10.5157/NEPS:SC4:10.0.o. Die Daten des NEPS wurden von 2008 bis 2013 als Teil des Rahmenprogramms zur Förderung der empirischen Bildungsforschung erhoben, welches vom Bundesministerium für Bildung und Forschung (BMBF) finanziert wurde. Seit 2014 wird NEPS vom Leibniz-Institut für Bildungsverläufe (LIfBi) an der Otto-FriedrichUniversität Bamberg in Kooperation mit einem deutschlandweiten Netzwerk weitergeführt. 
rinnen und Schüler mit sonderpädagogischem Förderbedarf umfasst der Datensatz 15239 Schülerinnen und Schüler aus 1012 Klassen der 9. Jahrgangsstufe (Welle 1 und 2). Die Stichprobenziehung der SC 4 basiert auf einem (multistage) cluster sampling (siehe Aßmann et al., 2019, für detaillierte Information). Die Klassenkompositionen, d.h. die Schätzungen der Anteile der Schülerinnen und Schüler in einer bestimmten Klasse mit einem bestimmten Merkmal (Akkulturationsprofil) unterliegen deshalb einer Ungenauigkeit, deren Ausmaß aus der Klassengröße (wie von der Lehrperson berichtet) und aus der Anzahl der Kinder einer Klasse, die tatsächlich befragt wurden, abgeleitet werden kann. Statistisch gesehen handelt es sich dabei um den Stichprobenfehler auf Klassenebene. Werden zum Beispiel in einer Klasse von 20 Kindern 17 Kinder (was in diesem Beispiel 85\% entspricht) befragt, und haben sieben dieser Schülerinnen und Schüler (oder 41\%) ein assimiliertes Akkulturationsprofil, beträgt der Stichprobenfehler 8\%, d.h. der wahre Anteil in dieser Klasse liegt mit 95\% Genauigkeit zwischen $33 \%$ und 49\%. Um genügend verlässliche Aussagen über den Zusammenhang der Klassenkomposition auf die individuelle Lesekompetenz treffen zu können, wurden für unsere Analysen diejenigen Klassen, bei denen jeweils die Gruppe der befragten Schülerinnen und Schüler bezüglich Klassenkomposition einen Stichprobenfehler von 10\% oder weniger aufwies, in die Stichprobe aufgenommen. Auf diese Weise wurden insgesamt 188 Klassen mit 4183 Schülerinnen und Schüler für die Mehrebenenanalysen ausgewählt. Der Definition des NEPS folgend hatten davon 1421 Schülerinnen und Schüler (35.6\%) einen Migrationshintergrund in erster, zweiter oder dritter Generation (vgl. Olczyk, Will \& Kristen, 2016). Das durchschnittliche Alter der Schülerinnen und Schüler beträgt 14.71 Jahre $(n=3908, M=14.71, S D=0.70$, $M i n=12$, $\operatorname{Max}=17)$.

\subsection{Instrumente und Variablen}

\subsubsection{Akkulturationsprofile}

Um die Akkulturationsorientierungen der Schülerinnen und Schüler mit Migrationshintergrund komplex zu erfassen, wurden in Anlehnung an das in Lilla et al. (2021) beschriebene Verfahren mit allen Schülerinnen und Schülern mit Migrationshintergrund in der Gesamtstichprobe vier latente Profile geschätzt. Tabelle 1 gibt einen Überblick über die Skalen, die berücksichtigt wurden, um affektive, behaviorale und kognitive Aspekte der Akkulturation abzudecken. Vor Durchführung der latenten Profilanalyse wurden alle Werte $z$-standardisiert. 
| Sebastian Thürer, Nanine Lilla, Wim Nieuwenboom \& Marianne Schüpbach

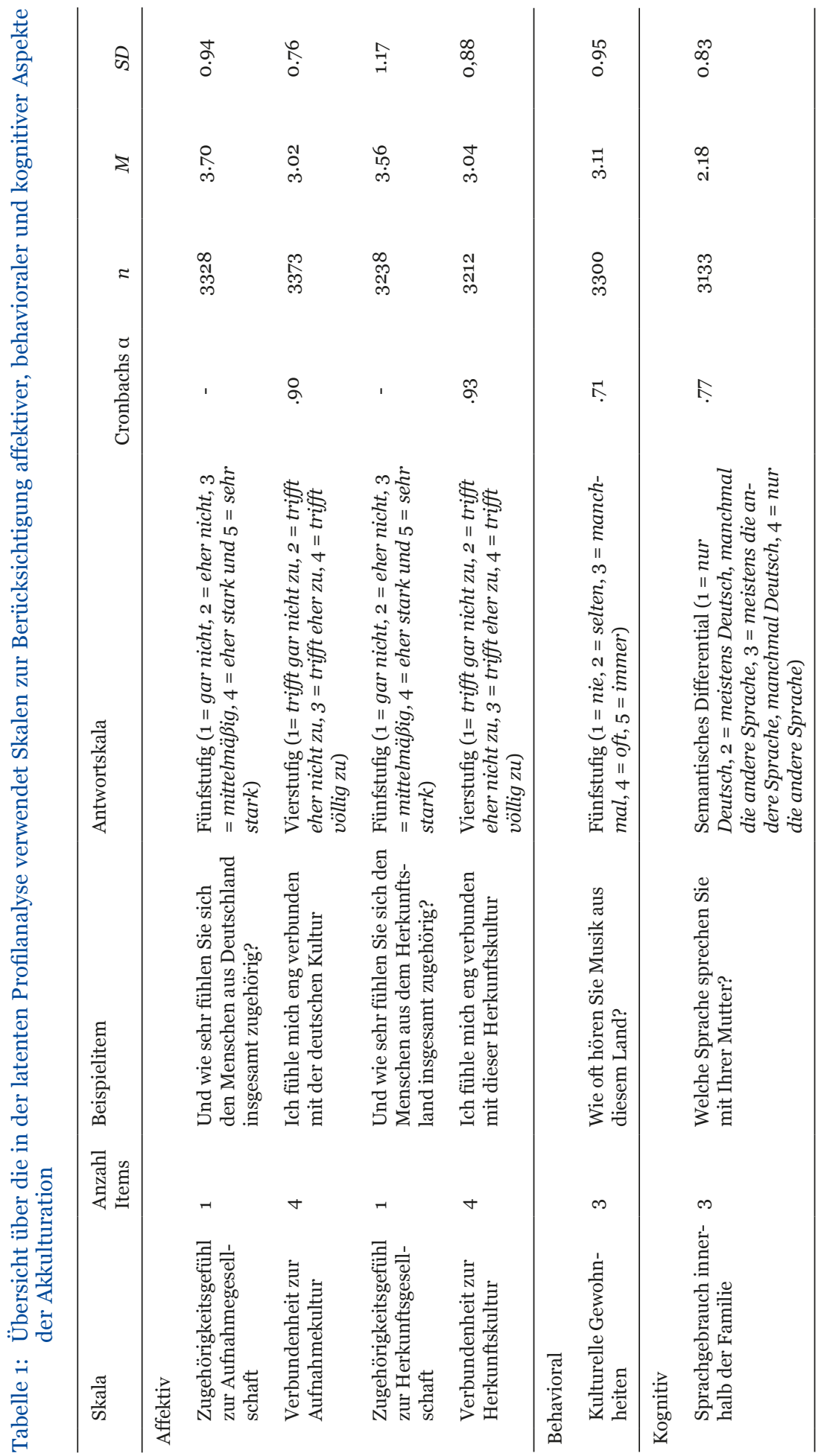


Abbildung 1 zeigt die in der Gesamtstichprobe mit 4070 Schülerinnen und Schülern mit Migrationshintergrund ermittelten latenten Profile. Die inhaltliche Interpretation der ermittelten Akkulturationsprofile ergibt ein assimiliertes, ein integriertes, ein separiertes und ein indifferentes Akkulturationsprofil.

Abbildung 1: Grafische Darstellung der vier Akkulturationsprofile.

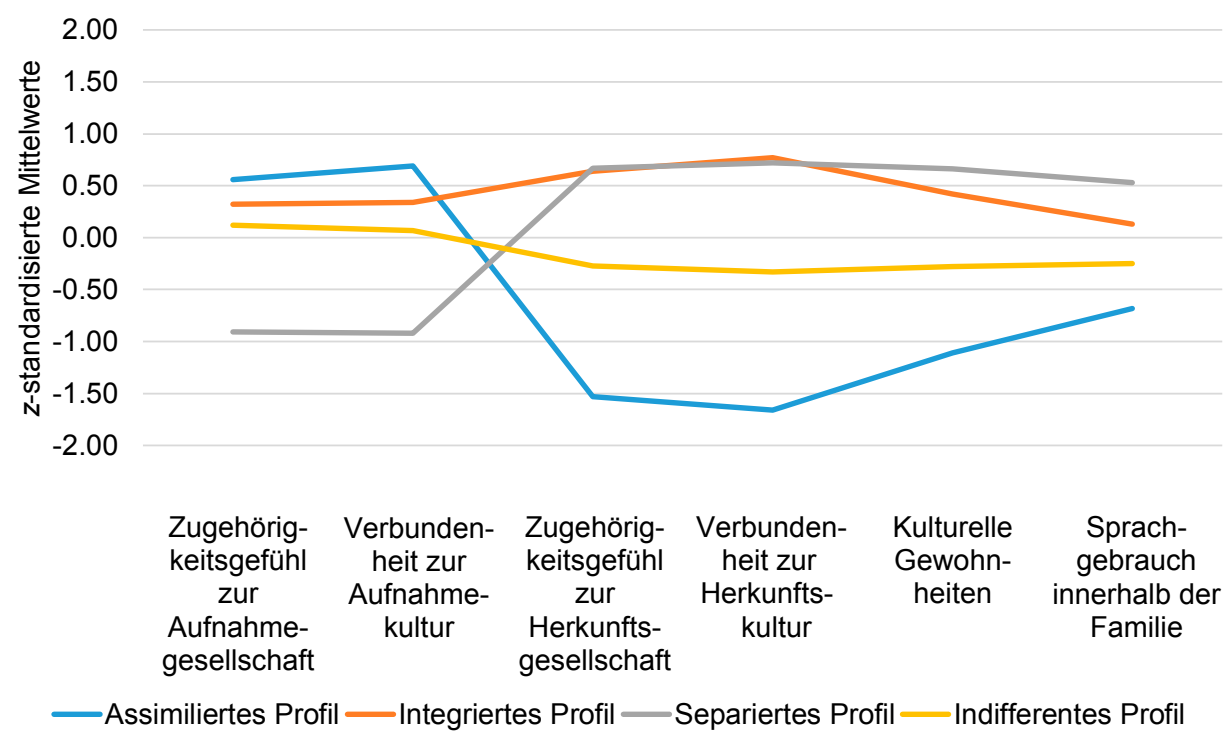

Das assimilierte Profil zeichnet sich bei den Zustimmungswerten zur Aufnahmekultur durch über dem Stichprobenmittelwert liegende Werte bei einem gleichzeitig unter dem Stichprobenmittelwert liegenden Wert zur Herkunftskultur aus. Auch die unter dem Stichprobenmittelwert liegenden Werte bei Fragen zur kulturellen Gewohnheit und zum Sprachgebrauch lassen den Schluss zu, dass die Herkunftskultur für diese Schülerinnen und Schüler eher eine untergeordnete Rolle spielt.

Im integrierten Profil werden durchgehend über dem Stichprobenmittelwert liegende Werte, sowohl bei den Fragen zur Aufnahme- wie auch zur Herkunftsgesellschaft und zur Aufnahme- und Herkunftskultur als auch bei den Fragen zur kulturellen Gewohnheit und dem Sprachgebrauch, verzeichnet.

Das separierte Profil zeichnet sich aus durch unter dem Stichprobenmittelwert liegende Werte bei den Fragen zur Aufnahmekultur und -gesellschaft und z.T. über dem Stichprobendurchschnitt liegenden Werten bei den Fragen zum Herkunftsland und -gesellschaft, zu kulturellen Gewohnheiten und zum Sprachgebrauch innerhalb der Familie. Dies lässt den Schluss zu, dass es sich hierbei um eine Gruppe von Schülerinnen und Schülern handelt, die, wie auch schon bei Berry (1997) beschrieben, sich dem Profil der kulturellen Separation zuordnen lassen. 
Das vierte Profil wird als indifferentes Akkulturationsprofil bezeichnet. Ausschlaggebend dafür ist die nicht eindeutige Tendenz der Orientierung. Die Werte liegen um den Stichprobenmittelwert.

\subsubsection{Verwendete Variablen in den Analysen}

Die Erfassung der Lesekompetenz basiert auf insgesamt 31 Items (Haberkorn, Pohl, Hardt \& Wiegand, 2012) und wurde in der Gesamtstichprobe als Weighted Maximum Likelihood Estimates (sog. WLE-Schätzer; Pohl \& Carstensen, 2012) ermittelt. In der Substichprobe der ausgewählten 188 Klassen weicht der Mittelwert minimal ab ( $n=3863$, Min $=-4.02, \operatorname{Max}=3.30, M=0.01, S D=1.25)$.

Als Proxy-Variable für den sozioökonomischen Status wurde das elterliche Bildungsniveau nach der International Standard Classification of Education (ISCED; Organisation for Economic Co-operation and Development [OECD], 1999) berücksichtigt. Dabei wurde der Elternteil mit dem höchsten Bildungsabschluss betrachtet $(n=2263, M=6.32, S D=2.60)$. Das Geschlecht der Schülerinnen $(n=1958$, $49.17 \%)$ und Schüler ( $n=2024,50.83 \%)$ sowie der besuchte Schultyp wurden als zusätzliche Kontrollvariablen berücksichtigt.

Tabelle 2 zeigt die Verteilung der Akkulturationsprofile sowie weiterer relevanter Merkmale in der ausgewählten Stichprobe.

\subsection{Statistisches Vorgehen}

Um die Lesekompetenz von Schülerinnen und Schülern mit Migrationshintergrund in Abhängigkeit von ihrem Akkulturationsprofil im Vergleich zur Lesekompetenz von Schülerinnen und Schülern ohne Migrationshintergrund unter Berücksichtigung der genesteten Datenstruktur (Schülerinnen und Schüler in Klassen) zu untersuchen, wurden Mehrebenenmodelle gerechnet, wobei die Akkulturationsprofile als Dummy-Variablen auf Individualebene modelliert und zusätzlich die Kontrollvariablen elterliches Bildungsniveau und Geschlecht auf Level 1 (Modell 1) sowie besuchter Schultyp auf Level 2 (Modell 2) berücksichtigt wurden. Darauf aufbauend wurde in Random-Intercept-Modellen der Zusammenhang zwischen den Anteilen der Akkulturationsprofile der Schülerinnen und Schüler mit Migrationshintergrund und der Lesekompetenz der Schülerinnen und Schüler der jeweiligen Klasse unter Berücksichtigung der Kontrollvariablen elterliches Bildungsniveau, Geschlecht auf Level 1 (Modell 3) sowie besuchter Schultyp auf Level 2 (Modell 4) geschätzt. Alle Berechnungen wurden mit Mplus Version 8.2 durchgeführt. Fehlende Werte wurden auf Basis von Full Information Maximum Likelihood ergänzt (Muthén \& Muthén, 1998-2017). 
Individuelle und im Klassenkontext vorherrschende Akkulturationsorientierung |

Tabelle 2: Verteilung der Stichprobenmerkmale auf Individual- und Klassenebene

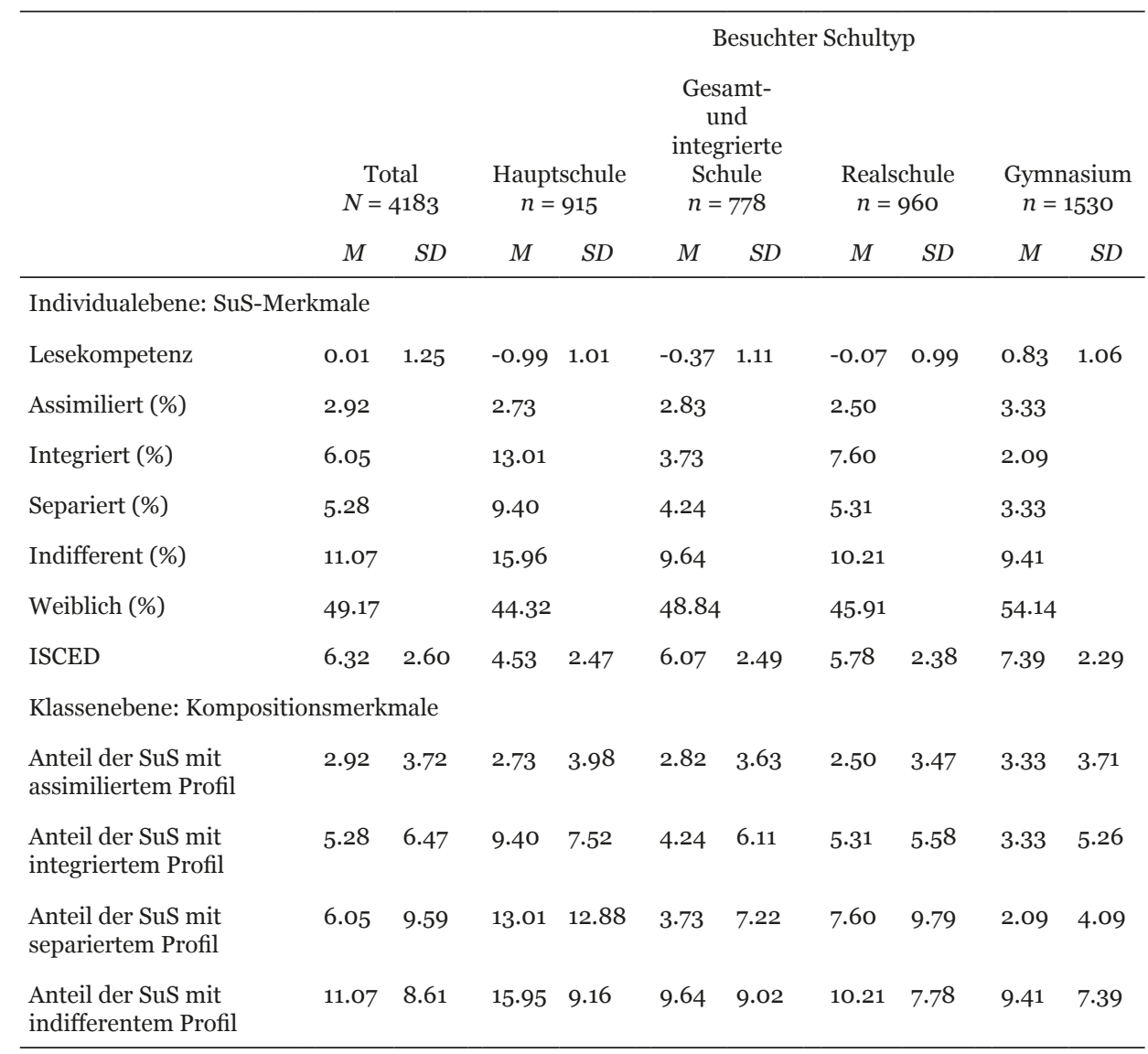

Anmerkung. SuS = Schülerinnen und Schüler. 


\section{Ergebnisse}

\subsection{Lesekompetenz von Schülerinnen und Schülern mit Migrationshintergrund in Abhängigkeit von ihrem Akkulturationsprofil im Vergleich zu Schülerinnen und Schülern ohne Migrationshintergrund}

Zur Schätzung, inwiefern Unterschiede zwischen Schülerinnen und Schülern ohne Migrationshintergrund und mit Migrationshintergrund in Abhängigkeit ihres Akkulturationsprofils bestehen, wurden die vier Akkulturationsprofile (Referenz: kein Migrationshintergrund) und die Kontrollvariablen des elterlichen Bildungshintergrundes und des Geschlechts (Referenz: männlich) aufgenommen. In Modell 1 zeigt sich bezüglich der abhängigen Variable, dass Schülerinnen und Schüler, die ein separiertes oder ein indifferentes Profil aufweisen, sich in ihrer Lesekompetenz signifikant von Schülerinnen und Schülern ohne Migrationshintergrund unterscheiden. Für Schülerinnen und Schüler mit einem assimilierten oder integrierten Profil zeigt sich hingegen kein signifikanter Unterschied zur Referenzgruppe. Schülerinnen und Schüler mit separiertem Profil haben im Vergleich zu Schülerinnen und Schülern ohne Migrationshintergrund eine deutlich niedrigere Lesekompetenz ( $B=-0.47, p<$.001). Ebenfalls zeigen Schülerinnen und Schüler mit indifferentem Profil $(B=-0.21, p<.001)$ eine niedrigere Lesekompetenz als Schülerinnen und Schüler ohne Migrationshintergrund.

Nach zusätzlicher Berücksichtigung des besuchten Schultyps auf Klassenebene (Referenz: Hauptschule) zeigt sich in Modell 2, dass weiterhin Unterschiede in der Lesekompetenz zwischen Schülerinnen und Schülern ohne Migrationshintergrund und Schülerinnen und Schülern mit Migrationshintergrund in Abhängigkeit ihres Akkulturationsprofils bestehen. Sowohl die Schülerinnen und Schüler mit separiertem Profil $(B=-0.44, p<.001)$ als auch mit indifferentem Profil $(B=-0.20$, $p<$.o01) weisen im Vergleich zur Referenzgruppe niedrigere Lesekompetenzen auf. Schülerinnen und Schüler mit assimiliertem oder integriertem Profil unterscheiden sich in ihrer Lesekompetenz auch bei Berücksichtigung des Schultyps nicht signifikant von Schülerinnen und Schülern ohne Migrationshintergrund.

\subsection{Zusammenhänge zwischen der Lesekompetenz und dem Anteil der Akkulturationsprofile in der Klasse als Merkmal der Klassenkomposition}

Zur Schätzung, inwiefern Zusammenhänge zwischen der Zusammensetzung einer Schulklasse im Hinblick auf die vorherrschenden Akkulturationsorientierungen der Schülerinnen und Schüler mit Migrationshintergrund und der individuellen Lesekompetenz aller Schülerinnen und Schüler bestehen, wurden die Anteile der vorherrschenden Akkulturationsprofile der Schülerinnen und Schüler mit Migrationshintergrund einer Klasse aggregiert und auf Klassenebene, zunächst ohne zu- 
Individuelle und im Klassenkontext vorherrschende Akkulturationsorientierung |

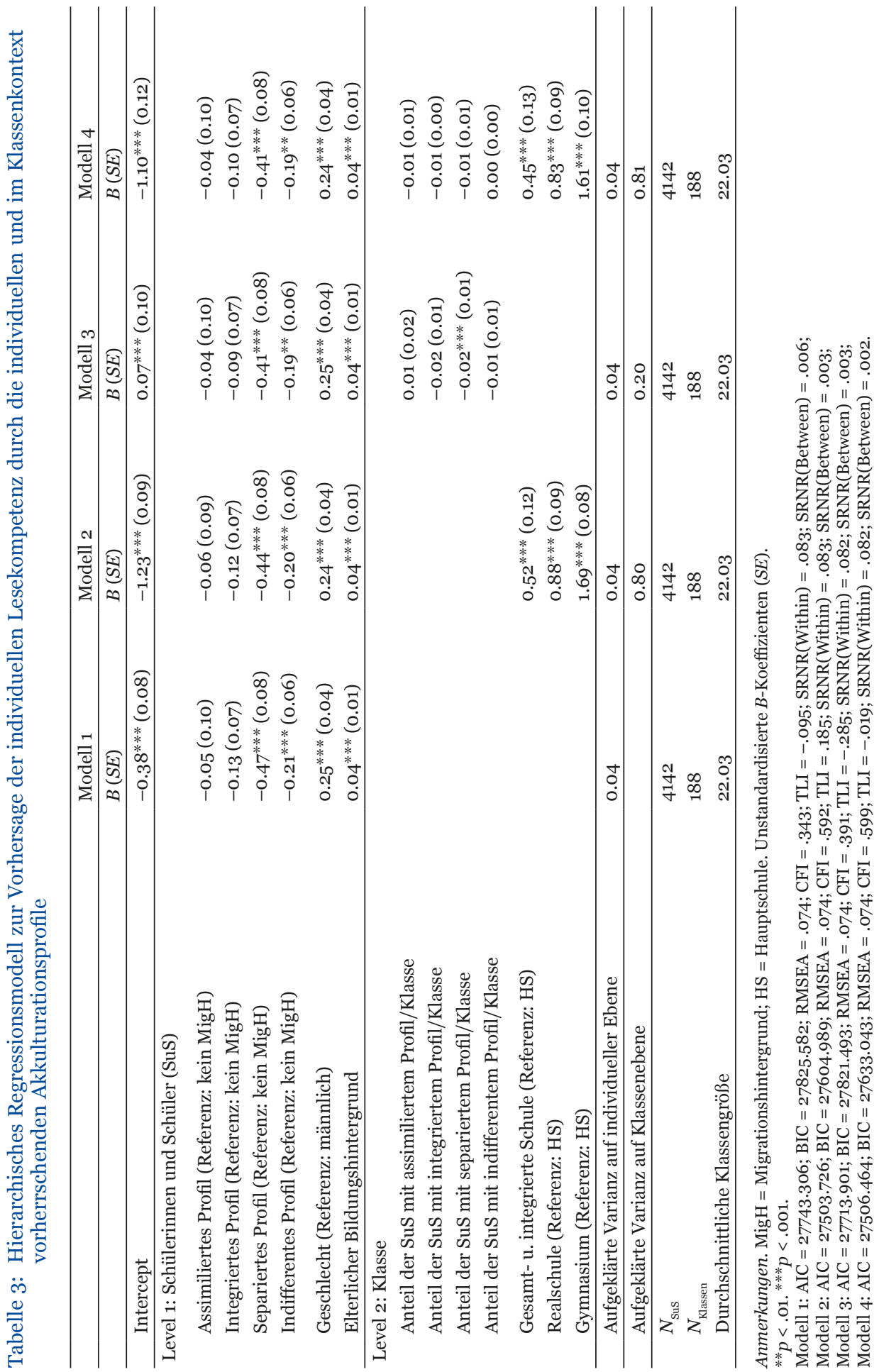


sätzliche Kontrolle des besuchten Schultyps, modelliert (Modell 3). Hierbei zeigt sich lediglich im Hinblick auf den Anteil der Schülerinnen und Schüler mit einem separierten Profil ein signifikanter Zusammenhang mit der Lesekompetenz: Je höher der Anteil der Schülerinnen und Schüler mit separiertem Akkulturationsprofil in der Klasse, desto geringer ist die individuelle Lesekompetenz der Schülerinnen und Schüler der Klasse $(B=-0.02, p<.001)$. Für die Anteile an Schülerinnen und Schülern mit assimiliertem, mit integriertem und mit indifferentem Akkulturationsprofil zeigen sich keine signifikanten Zusammenhänge mit der erreichten Lesekompetenz der Schülerinnen und Schüler.

Nach zusätzlicher Berücksichtigung des besuchten Schultyps auf Klassenebene (Modell 4) zeigen sich keine signifikanten Zusammenhänge zwischen den Anteilen der verschiedenen Akkulturationsprofile auf Klassenebene und der individuellen Lesekompetenz der Schülerinnen und Schüler. Die Koeffizienten der als zusätzliche Prädiktoren auf Klassenebene eingeführten Schultypen-Dummies zeigen vergleichbar wie im Modell 2 auf, dass ein starker Zusammenhang zwischen der besuchten Schulform und der erreichten Lesekompetenz der Schülerinnen und Schüler besteht.

\section{Diskussion}

Ausgangspunkt dieses Beitrags war die persistierende Bildungsungleichheit zwischen Schülerinnen und Schülern mit und ohne Migrationshintergrund und die Erkenntnis, dass unterschiedliche Akkulturationsorientierungen differentiell mit der schulischen Leistung von Schülerinnen und Schülern mit Migrationshintergrund in Zusammenhang stehen.

Darauf aufbauend wurde mit der ersten Fragestellung untersucht, inwiefern sich die Lesekompetenzen von Schülerinnen und Schülern mit Migrationshintergrund in Abhängigkeit von ihrer Akkulturationsorientierung von der erreichten Lesekompetenz von Schülerinnen und Schülern ohne Migrationshintergrund unterscheiden. Um Akkulturationsorientierungen komplex zu erfassen, wurden mit allen Schülerinnen und Schülern mit Migrationshintergrund aus dem Gesamtdatensatz der Startkohorte 4 des NEPS unter Berücksichtigung affektiver, behavioraler und kognitiver Aspekte von Akkulturation anhand einer latenten Profilanalyse vier distinkte Akkulturationsprofile identifiziert. Mit Daten aus 188 ausgewählten Klassen wurden anschließend Mehrebenenmodelle gerechnet. Die vorliegenden Ergebnisse zeigen signifikante Unterschiede in der individuellen Lesekompetenz zwischen Schülerinnen und Schülern mit separiertem sowie indifferentem Akkulturationsprofil im Vergleich zu Schülerinnen und Schülern ohne Migrationshintergrund. Insbesondere Schülerinnen und Schüler mit separiertem Akkulturationsprofil, aber auch solche mit indifferentem Akkulturationsprofil, haben deutlich niedrigere Lesekompetenzen als Schülerinnen und Schüler ohne Migrationshintergrund. Für Schülerinnen und Schüler mit assimiliertem und integriertem Akkulturationsprofil zeigen sich hingegen keine signifikanten Unterschiede verglichen mit Schülerin- 
nen und Schülern ohne Migrationshintergrund. Dieses Befundmuster bleibt nahezu unverändert, wenn die von den Schülerinnen und Schülern besuchten Schulformen berücksichtigt werden, die, wie die Koeffizienten zeigen, sehr starke Prädiktoren der individuellen Lesekompetenz darstellen. Die geringeren Lesekompetenzen von Schülerinnen und Schülern mit separiertem und indifferentem Akkulturationsprofil stehen teilweise im Einklang mit vorherigen Befunden. Die von Edele et al. (2013) gezeigte höhere Lesekompetenz von assimilierten Schülerinnen und Schülern im Vergleich zu den Schülerinnen und Schülern ohne Migrationshintergrund ließ sich in unserer Studie jedoch nicht replizieren. Möglicherweise ist dies zurückzuführen auf die Beachtung der genesteten Datenstruktur, bei der angenommen werden muss, dass die Lesekompetenzen von Schülerinnen und Schülern innerhalb einer Klasse weniger stark variieren als über Klassen hinweg. Eine weitere mögliche Begründung könnte der unterschiedliche Umgang mit konfundierten Variablen und das Vorgehen bei der Operationalisierung der Akkulturationsorientierungen darstellen. Während Edele et al. (2013) Akkulturationsorientierungen über Mediansplit lediglich zweier affektiv-identifikativer Variablen erfassten und im Regressionsmodell Variablen wie beispielsweise die Familiensprache kontrollierten, konnten wir durch die Anwendung einer latenten Profilanalyse die in der Familie gesprochene Sprache sowie weitere Aspekte bereits bei der Operationalisierung der Akkulturationsorientierungen berücksichtigen. Wie auch in anderen Studien (Hannover et al., 2013; Schotte et al., 2018) gezeigt, scheint im deutschen Schulkontext vor allem die Orientierung an der Aufnahmegesellschaft, die in unserer Studie sowohl für Schülerinnen und Schüler mit assimiliertem als auch für Schülerinnen und Schüler mit integriertem Profil ausgeprägt ist, relevant für Bildungserfolg. Die Unterschiede im Sprachgebrauch innerhalb der Familie zwischen assimiliertem und integriertem Akkulturationsprofil kommen in unseren Analysen hinsichtlich der Lesekompetenz der Schülerinnen und Schüler nicht zum Tragen. Im Unterschied zu bisheriger Forschung ergab die latente Profilanalyse ein Akkulturationsprofil, welches aufgrund seiner Ausprägungen im Hinblick auf die Orientierung an der Aufnahme- und Herkunftskultur als indifferent bezeichnet wurde. Obwohl sich dieses Profil hinsichtlich der Orientierung an der Aufnahmekultur als vergleichbar mit dem integrierten Profil zeigt, haben Schülerinnen und Schüler mit diesem Akkulturationsprofil geringere Lesekompetenzen als Schülerinnen und Schüler ohne Migrationshintergrund. Dabei handelt es sich mit $44 \%$ um beinahe jede zweite Schülerin und jeden zweiten Schüler mit Migrationshintergrund in der Stichprobe. Zukünftige Studien sollten daher prüfen, ob sich dieses Profil und seine hohe Prävalenz replizieren lassen.

Aufgrund des querschnittlichen Designs unserer Studie lassen sich die Befunde nicht kausal interpretieren. Das heißt, es ist sowohl denkbar, dass die Lesekompetenz von Schülerinnen und Schülern mit Migrationshintergrund beeinflusst wird von ihren Akkulturationsorientierungen und den damit einhergehenden Ressourcen, als auch, dass die Lesekompetenz einer Schülerin bzw. eines Schülers mit Migrationshintergrund die präferierte Akkulturationsorientierung bedingt. Darüber hinaus wäre auch möglich, dass sich die beiden Wirkrichtungen nicht gegenseitig 
ausschließen, sondern wie in einer Art Kreislauf nebeneinanderher existieren. Nach unserem Kenntnisstand gibt es zur Frage nach der Kausalität bislang kein empirisches Wissen. Mittels Daten des Nationalen Bildungspanels wäre eine längsschnittliche Betrachtung dieser Zusammenhänge grundsätzlich möglich, aufgrund fehlender Messzeitpunkte in den relevanten Variablen aber mit erheblichen Limitationen verbunden.

Zusammengefasst stützen die Ergebnisse jedenfalls die Annahme, dass nicht der Migrationshintergrund per se für ein schlechteres Abschneiden beim Erwerb von Lesekompetenzen verantwortlich gemacht werden kann, und verdeutlichen, dass die grobe Kategorisierung in Schülerinnen und Schüler mit und ohne Migrationshintergrund im Hinblick auf schulische Ungleichheit wenig zielführend ist.

Ähnlich dieser Erkenntnis zeigt der nationale Forschungsstand zu Kompositionseffekten im Hinblick auf die schulische Leistung der Schülerinnen und Schüler, dass der Anteil der Schülerinnen und Schüler mit Migrationshintergrund in einer Schulklasse keinen spezifischen Einfluss hat (vgl. Dumont et al., 2013), wohl aber damit in Zusammenhang stehende Variablen wie beispielsweise die Erstsprache der Schülerinnen und Schüler mit Migrationshintergrund (Seuring et al., 2020). Aufbauend auf dieser Erkenntnis wurde mit der zweiten Forschungsfrage untersucht, inwiefern Zusammenhänge zwischen den auf Klassenebene vorherrschenden Akkulturationsprofilen und der individuellen Leseleistung der Schülerinnen und Schüler bestehen. Die Ergebnisse unserer Analyse zeigen einen einzigen signifikanten Zusammenhang zwischen dem Anteil der Schülerinnen und Schüler mit separiertem Akkulturationsprofil in der Klasse und der Lesekompetenz der Schülerinnen und Schüler: Je höher der Anteil der Schülerinnen und Schüler mit diesem Profil in der Klasse, desto niedriger ist die individuelle Lesekompetenz aller Schülerinnen und Schüler der jeweiligen Klasse. Allerdings ist der negative Zusammenhang nur schwach und nach Kontrolle des besuchten Schultyps nicht mehr signifikant. Wesentlich bedeutsamer erscheint, wie auch in anderen Studien gezeigt, die besuchte Schulform (z. B. Baumert, Stanat \& Watermann, 2006). Eine Limitation ergibt sich aufgrund des Samplings der NEPS-Datenerhebung, bei der Schulklassen nicht immer vollständig erhoben wurden. Unsere Analysen wurden daher nur mit einer Substichprobe berechnet, für die ausreichend Information über die Klassenkomposition verfügbar war.

Insgesamt lässt sich resümieren, dass sich für das deutsche Schulsystem eine starke Orientierung an der Aufnahmegesellschaft, wie im assimilierten und integrierten Akkulturationsprofil der Fall, als vorteilhaft in Bezug auf die Lesekompetenz von Schülerinnen und Schülern mit Migrationshintergrund erweist. In zukünftigen Untersuchungen zur Bildungsungleichheit sollten die Akkulturationsorientierungen der Schülerinnen und Schüler mit Migrationshintergrund stärker in den Blick genommen werden. Von besonderem Interesse wäre hierbei, der Frage nachzugehen, worin die anscheinend problematische Distanz zur deutschen Kultur und deutschen Gesellschaft begründet liegt: Wollen oder können sich diese Schülerinnen und Schüler nicht stärker am Aufnahmeland orientieren? Ist eine separierte Akkulturationsorientierung möglicherweise Folge schwächerer schulischer 
Leistung? Und falls dies zuträfe, wie könnte das durch unterrichtliches Handeln verhindert werden?

\section{Acknowledgments}

Der Beitrag wurde gefördert durch die Deutsche Forschungsgemeinschaft (DFG) Projektnummer: LI 3067-1/1.

\section{Literatur}

Aßmann, C., Steinhauer, H. W., Würbach, A., Zinn, S., Hammon, A., Kiesl, H. ... Blossfeld, H.-P. (2019). Sampling designs of the National Educational Panel Study: Setup and panel development. In H.-P. Blossfeld \& H.-G. Roßbach (Hrsg.), Education as a lifelong process (Bd. 3, S. 35-55). Springer VS. https://doi. org/10.1007/978-3-658-23162-0_3

Autorengruppe Bildungsberichterstattung. (2018). Bildung in Deutschland 2018: Ein indikatorengestützter Bericht mit einer Analyse zu Bildung und Migration. wbv. https://doi.org/10.3278/6001820fw

Baumert, J., Stanat, P. \& Watermann, R. (Hrsg.). (2006). Herkunftsbedingte Disparitäten im Bildungswesen: Differenzielle Bildungsprozesse und Probleme der Verteilungsgerechtigkeit. Vertiefende Analysen im Rahmen von PISA 2000. VS Verlag für Sozialwissenschaften. https://doi.org/10.1007/978-3-531-90082-7

Becker, R. (Hrsg.). (2011). Integration durch Bildung. Bildungserwerb von jungen Migranten in Deutschland. VS Verlag für Sozialwissenschaften. https://doi. org/10.1007/978-3-531-93232-3

Berry, J. (1997). Immigration, acculturation, and adaptation. Applied Psychology, 46(1), 5-34. https://doi.org/10.1111/j.1464-0597.1997.tb01087.x

Berry, J. \& Hou, F. (2016). Immigrant acculturation and wellbeing in Canada. Canadian Psychology/Psychologie canadienne, 57(4), 254-264. https://doi.org/10.1037/ capooooo64

Berry, J., Kim, U., Power, S., Young, M. \& Bujaki, M. (1989). Acculturation attitudes in plural societies. Applied Psychology, 38(2), 185-206. https://doi. org/10.1111/j.1464-0597.1989.tb01208.x

Berry, J., Phinney, J. S., Sam, D. L. \& Vedder, P. (Hrsg.). (2006). Immigrant youth in cultural transition. Acculturation, identity, and adaptation across national contexts. Erlbaum. https://doi.org/10.4324/9780415963619

Blossfeld, H.-P., Roßbach, H.-G. \& von Maurice, J. (Hrsg.). (2011). Education as a lifelong process: The German National Educational Panel Study (NEPS) (Zeitschrift für Erziehungswissenschaft: Sonderheft 14). VS Verlag für Sozialwissenschaften.

Boudon, R. (1974). Education, opportunity, and social inequality: Changing prospects in Western society. Wiley.

Celeste, L., Meeussen, L., Verschueren, K. \& Phalet, K. (2016). Minority acculturation and peer rejection: Costs of acculturation misfit with peer-group norms. British Journal of Social Psychology, 55(3), 544-563. https://doi.org/10.1111/bjso.12147

Driessen, G. (2002). School composition and achievement in primary education: A large-scale multilevel approach. Studies in Educational Evaluation, 28(4), 347368. https://doi.org/10.1016/So191-491X(02)00043-3

Dumont, H., Maaz, K., Neumann, M. \& Becker, M. (2014). Soziale Ungleichheiten beim Übergang von der Grundschule in die Sekundarstufe I: Theorie, Forschungsstand, Interventions- und Fördermöglichkeiten. Zeitschrift für Erziehungswissenschaft, 17(S2), 141-165. https://doi.org/10.1007/s11618-013-0466-1 
Dumont, H., Neumann, M., Maaz, K. \& Trautwein, U. (2013). Die Zusammensetzung der Schülerschaft als Einflussfaktor für Schulleistungen. Psychologie in Erziehung und Unterricht, 6o(3), 163-183. https://doi.org/10.2378/peu2013.art14d

Edele, A., Stanat, P., Radmann, S. \& Segeritz, M. (2013). Kulturelle Identität und Lesekompetenz von Jugendlichen aus zugewanderten Familien. In N. Jude \& E. Klieme (Hrsg.), PISA 2009 - Impulse für die Schul- und Unterrichtsforschung (Zeitschrift für Pädagogik Beiheft, Bd. 59, S. 84-110). Beltz Juventa.

Fox, R. S., Merz, E. L., Solórzano, M. T. \& Roesch, S. C. (2013). Further examining Berry's model: The applicability of latent profile analysis to acculturation. Measurement and evaluation in counseling and development: The official publication of the Association for Measurement and Evaluation in Counseling and Development, 46(4), 270-288. https://doi.org/10.1177/0748175613497036

Haberkorn, K., Pohl, S., Hardt, K. \& Wiegand, E. (2012). NEPS technical report for reading - Scaling results of starting cohort 4 in ninth grade. (NEPS Working Papers No. 16). Otto-Friedrich-Universität Bamberg, Nationales Bildungspanel. https://doi.org/10.5157/NEPS:WP16:1.0

Hannover, B., Morf, C. C., Neuhaus, J., Rau, M., Wolfgramm, C. \& Zander-Musić, L. (2013). How immigrant adolescents' self-views in school and family context relate to academic success in Germany. Journal of Applied Social Psychology, 43(1), 175-189. https://doi.org/10.1111/j.1559-1816.2012.00991.x

Jugert, P., Pink, S., Fleischmann, F. \& Leszczensky, L. (2020). Changes in Turkishand Resettler-origin adolescents' acculturation profiles of identification: A threeyear longitudinal study from Germany. Journal of Youth and Adolescence, 49(12), 2476-2494. https://doi.org/10.1007/s10964-020-01250-w

Lilla, N., Thürer, S., Nieuwenboom, W. \& Schüpbach, M. (2021). Assimiliert - Abitur, separiert - Hauptschulabschluss? Zum Zusammenhang zwischen Akkulturation und angestrebtem Schulabschluss. Zeitschrift für Erziehungswissenschaft, 24, 571-592. https://doi.org/10.1007/s11618-021-01004-9

Makarova, E. \& Birman, D. (2015). Cultural transition and academic achievement of students from ethnic minority backgrounds: A content analysis of empirical research on acculturation. Educational Research, 57(3), 305-330. https://doi.org/ 10.1080/00131881.2015.1058099

Matsunaga, M., Hecht, M. L., Elek, E. \& Ndiaye, K. (2010). Ethnic identity development and acculturation. A longitudinal analysis of Mexican-heritage youth in the southwest United States. Journal of Cross-Cultural Psychology, 41(3), 410-427. https://doi.org/10.1177/0022022109359689

Muthén, L. K. \& Muthén, B. O. (1998-2017). Mplus. Statistical analysis with latent variables. User's guide (8. Aufl.). Muthén \& Muthén.

Nieri, T., Lee, C., Kulis, S. \& Marsiglia, F. F. (2011). Acculturation among Mexicanheritage preadolescents: A latent class analysis. Social Science Research, 4O(4), 1236-1248. https://doi.org/10.1016/j.ssresearch.2011.02.005

Olczyk, M., Will, G. \& Kristen, C. (2016). Immigrants in the NEPS: Identifying generation status and group of origin (NEPS Survey Papers No. 41a, 2. Aufl.). Leibniz Institute for Educational Trajectories, National Educational Panel Study. https:// doi.org/10.5157/NEPS:SP04:1.0

Organisation for Economic Co-operation and Development. (1999). Classifying educational programmes: Manual for ISCED-97 implementation in OECD countries. OECD.

Pohl, S. \& Carstensen, C. H. (2012). NEPS technical report - Scaling the data of the competence tests (NEPS Working Papers No. 14). Otto-Friedrich-Universität Bamberg, Nationales Bildungspanel. https://doi.org/10.5157/NEPS:WP14:1.0

Schotte, K., Stanat, P. \& Edele, A. (2018). Is integration always most adaptive? The role of cultural identity in academic achievement and in psychological adaptation of 
immigrant students in Germany. Journal of Youth and Adolescence, 47(1), 16-37. https://doi.org/10.1007/s10964-017-0737-x

Schwartz, S. J., Unger, J. B., Zamboanga, B. L. \& Szapocznik, J. (2010). Rethinking the concept of acculturation: Implications for theory and research. American Psychologist, 65(4), 237-251. https://doi.org/10.1037/a0019330

Schwartz, S. J. \& Zamboanga, B. L. (2008). Testing Berry's model of acculturation: A confirmatory latent class approach. Cultural Diversity and Ethnic Minority Psychology, 14(4), 275-285. https://doi.org/10.1037/ao012818

Seuring, J., Rjosk, C. \& Stanat, P. (2020). Ethnic classroom composition and minority language use among classmates: Do peers matter for students' language achievement? European Sociological Review, 36(6), 920-936. https://doi.org/10.1093/ esr/jcaaO22

Stanat, P. (2006). Schulleistungen von Jugendlichen mit Migrationshintergrund: Die Rolle der Zusammensetzung der Schülerschaft. In J. Baumert, P. Stanat \& R. Watermann (Hrsg.), Herkunftsbedingte Disparitäten im Bildungswesen: Differenzielle Bildungsprozesse und Probleme der Verteilungsgerechtigkeit. Vertiefende Analysen im Rahmen von PISA 2000 (S. 189-219). VS Verlag für Sozialwissenschaften. https://doi.org/10.1007/978-3-531-90082-7_5

Stanat, P., Schwippert, K. \& Gröhlich, C. (2010). Der Einfluss des Migrantenanteils in Schulklassen auf den Kompetenzerwerb. Längsschnittliche Überprüfung eines umstrittenen Effekts. In C. Allemann-Ghionda, P. Stanat, K. Göbel \& C. Röhner (Hrsg.), Migration, Identität, Sprache und Bildungserfolg. Längsschnittliche Überprüfung eines umstrittenen Effekts (S. 147-165). Beltz.

Ward, C. (1996). Acculturation. In D. Landis \& R. S. Bhagat (Hrsg.), Handbook of intercultural training (2. Aufl., S. 124-147). Sage Periodicals Press.

Ward, C. (2001). The A, B, Cs of acculturation. In D. R. Matsumoto (Hrsg.), The handbook of culture and psychology (S. 411-445). Oxford University Press.

Weis, M., Müller, K., Mang, J., Heine, J.-H., Mahler, N. \& Reiss, K. (2019). Soziale Herkunft, Zuwanderungshintergrund und Lesekompetenz. In K. Reiss, M. Weis, E. Klieme \& O. Köller (Hrsg.), PISA 2018. Grundbildung im internationalen Vergleich (S. 129-162). Waxmann. 